\title{
Preoperative Weight Loss: A Component of the Preoperative Program in Bariatric Surgery
}

\author{
Marinus Wiezer • Ignace Jansen • Anders Thorell
}

Received: 21 June 2009 / Accepted: 22 September 2009 /Published online: 15 October 2009

(C) Springer Science + Business Media, LLC 2009

To the editor:

With interest we read the letter to the editor by Kalarchian and Marcus [1] regarding the value of preoperative weight loss in bariatric surgery. In their editorial, Tarnoff et al. [2] give a good summary of the literature on preoperative weight loss in bariatric surgery and concluded that the question as to whether it is time to include preoperative weight loss as a component in the preoperative preparation process remains to be fully answered. As pointed out by Kalarchian and Marcus [1], there is unfortunately little evidence regarding how much weight loss is required to obtain the mentioned benefits. Furthermore, it still needs to be established which regimen or diet is best in order to achieve the preoperative weight loss.

Kalarchian and Markus are investigating an empirically supported 6-month behavioral weight management program. They anticipate that this will lead to fewer surgical complications and an enhancement of postoperative weight loss. We agree that a preoperative educational program may lead to a better postoperative compliance and better longterm results. However, for this group of patients, 6 months seems to be a very long period, and it could be questioned whether this is necessary, at least if the goal is to reduce surgical complications.

\section{Wiezer $(\bowtie)$}

Department of Surgery, Sint Antonius Ziekenhuis,

Postbus 2500, 3430 EM, Nieuwegein, The Netherlands

e-mail: r.wiezer@antonius.net

I. Jansen

Department of Surgery, Rijnstate Hospital,

Arnhem, The Netherlands

A. Thorell

Karolinska Institutet, Department of Clinical Science,

Danderyd Hospital \& Department of Surgery, Ersta Hospital,

11691 Stockholm, Sweden
Thus, by serial imaging, Colles et al. [3] demonstrated that intake of a very low-energy diet (VLED) significantly reduces liver and abdominal fat by approximately $18.7 \%$ over a period of 6 weeks. Interestingly, $80 \%$ of the decrease in liver volume was achieved within the first 2 weeks of VLED.

We experience that in line with this finding, an increasing number of bariatric surgical centers routinely recommend a 2- to 3-week period of low-calorie diets or very low-calorie diets, assuming that this routine will reduce the risk of surgical complications. However, this has never been evaluated properly in high-volume centers with operating times in the range of 50-120 $\mathrm{min}$. Therefore, we are currently conducting a multicenter prospective randomized single blinded study to evaluate the effect of short-term preoperative weight loss using VLED on operative outcome (trial registration no. CT20090015; www.kctr.se).

This study was designed by participants of the "European Obesity Academy" (http://www.eoa.nu/). In this program, young specialists join together with experienced senior consultants and scientists in the field of obesity in research and training, with participants in most European countries. We intend to present the results of this study during The International Congress on Obesity in Stockholm, July 2010.

With this study, we hope to contribute to the ongoing research with the aim of optimizing our surgical care for the obese patient.

\section{References}

1. Kalarchian MA, Marcus MD. Preoperative weight loss in bariatric surgery. Letter to the editor. Obes Surg. 2009;19:539.

2. Tarnoff M, Kaplan LM, Sikora S. An evidenced-based assessment of preoperative weight loss in bariatric surgery. Obes Surg. 2008;18:1059-61.

3. Colles SL, Dixon JB, Marks P, et al. Preoperative weight loss with a very-low-energy diet: quantitation of changes in liver and abdominal fat by serial imaging. Am J Clin Nutr. 2006;84:304-11. 